\title{
Evaluation of abnormal iron distribution in specific regions in the brains of patients with Parkinson's disease using quantitative susceptibility mapping and $\mathrm{R}^{*}$ mapping
}

\author{
QINGQING CHENG $^{1 *}$, JIAXI HUANG ${ }^{1 *}$, JIANYE LIANG $^{1}$, MENGJIE MA $^{1}$, QIAN ZHAO ${ }^{2}$, \\ XUEPING LEI $^{3}$, CHANGZHENG SHI $^{1}$ and LIANGPING LUO ${ }^{1}$ \\ ${ }^{1}$ Medical Imaging Center, The First Affiliated Hospital of Jinan University, Guangzhou, Guangdong 510630; \\ ${ }^{2}$ School of Public Health, Guangzhou Medical University, Guangzhou, Guangdong 511436; \\ ${ }^{3}$ Key Laboratory of Molecular Target and Clinical Pharmacology, \\ School of Pharmaceutical Sciences and Fifth Affiliated Hospital, Guangzhou Medical University, \\ Guangzhou, Guangdong 510799, P.R. China
}

Received July 30, 2019; Accepted February 28, 2020

DOI: $10.3892 / \mathrm{etm} .2020 .8645$

\begin{abstract}
The primary aim of the present study was to evaluate abnormal iron distribution in specific regions of the brains in patients with Parkinson's disease (PD) using quantitative susceptibility mapping (QSM) and R2* mapping, and to compare the diagnostic performances of QSM and $\mathrm{R} 2 *$ mapping in differentiating patients with PD with that in normal controls. A total of 25 patients with idiopathic PD and 28 sex-and age-matched normal controls were included in the present study and their brains investigated using a 3T scanner. Magnetic resonance imaging techniques, namely, QSM and R2* mapping, were applied to generate susceptibility and $\mathrm{R} 2^{*}$ values. The differences in susceptibility and R2* values in deep grey matter nuclei between patients with PD and the normal controls were compared using independent samples t-tests. The abilities of QSM and $\mathrm{R} 2^{*}$ mapping to classify patients with $\mathrm{PD}$ and normal controls were analyzed using receiver operating characteristic curves. Correlation analyses between imaging parameters (e.g. susceptibility and R2* values) and clinical feature (disease severity assessed using the Hoehn and Yahr score) were performed. The intra-class correlation coefficient (ICC) for susceptibility (ICC $=0.977 ; \mathrm{P}<0.001)$ and $\mathrm{R} 2 *(\mathrm{ICC}=0.945 ; \mathrm{P}<0.001)$ values between two neuro-radiologists were $>0.81$, showing excellent
\end{abstract}

Correspondence to: Professor Liangping Luo or Professor Changzheng Shi, Medical Imaging Center, The First Affiliated Hospital of Jinan University, 613 Huangpu Road West, Guangzhou, Guangdong 510630, P.R. China

E-mail: tluolp@jnu.edu.cn

E-mail: tsczcn@jnu.edu.cn

*Contributed equally

Key words: Parkinson's disease, iron, magnetic resonance imaging, quantitative susceptibility mapping, $\mathrm{R} 2 *$ mapping inter-rater agreement. The susceptibility values were significantly increased in the substantia nigra (SN) and red nucleus, but were decreased in the putamen of patients with PD compared with that in the corresponding brain regions of normal controls. However, increased R2* values were observed only in the $\mathrm{SN}$ in patients with PD. QSM showed higher sensitivity and specificity compared with $\mathrm{R}^{*}$ mapping to separate the patients with PD from the normal controls. There were no significant correlations between the susceptibility/R2* values and clinical features in all targeted regions of the brains in patients with PD. In conclusion, both QSM and R2* mapping are feasible to calculate the iron levels in human brains, and QSM provides a more sensitive and accurate method to assess regional abnormal iron distribution in patients with PD.

\section{Introduction}

Parkinson's disease (PD) is a common neurodegenerative disease whose primary clinical characteristics include tremor, bradykinesia, muscle rigidity and abnormal posture and pace (1). Approximately $1 \%$ of people over 60 years old in industrialized countries have PD (2). The selective depletion of dopaminergic neurones in the substantia nigra (SN) (3), the primary region of the brain that is impaired in $\mathrm{PD}$, the presence of $\alpha$-synuclein containing Lewy bodies and increased iron deposition in the $\mathrm{SN}$, are the most common pathological features of PD $(4,5)$. However, the role of increased iron deposition in the pathogenesis of PD is unclear and increased iron deposition may be a cause or by-product of cellular death or dysfunction in PD (6). A recent pooled iron-related gene study suggested that increased iron deposition in PD-related brain regions such as SN played a role in the aetiology of PD (7). Sian-Hulsmann et al (8) and Andersen (9) reported that iron-mediated cellular destruction is mediated primarily via reactive oxygen and/or nitrogen species-induced oxidative stress, which can induce neuronal vulnerability.

At present, the diagnosis of PD is based on the presence of motor symptoms and a set of clinical evaluations [for example, 
Unified Parkinson's Disease Rating Scale (UPDRS)], but the lack of objective and accessible diagnostic indicators may lead to missed diagnosis, misdiagnosis and delayed treatment $(1,10)$. As a result, objective, accessible and non-invasive diagnostic biomarkers are required. Several magnetic resonance imaging (MRI) techniques, such as transverse relaxation rate (R2), effective transverse relaxation rate $\left(\mathrm{R} 2^{*}\right)$ and phase imaging, have been used to evaluate iron content in the brain, as the direct measurement of cerebral iron content in vivo is not feasible. Hardy et al (11) compared the R2 and the tissue iron concentration in selected grey matter regions of the brains of 12 female rhesus monkeys. Their results indicated that R2 was highly correlated with the total iron concentration and that this correlation appeared to depend upon the iron concentration. Aquino et al (12) determined the values of iron accumulation in the basal ganglia of healthy volunteers of different ages (range, 1-80 years) using $\mathrm{R} 2 *$ mapping and good correlation coefficients were found for GP $\left(R^{2}=0.64\right)$, PT $\left(R^{2}=0.51\right)$, and SN $\left(R^{2}=0.53\right)$ when compared with findings in a previous post-mortem study (13). A multi-center study was performed to assess the inter-scanner and inter-subject variability of $\mathrm{R} 2^{*}$ mapping. The results indicated that $\mathrm{R} 2^{*}$ is a robust and reproducible measure in a multi-center setting provided that a standardized MRI protocol is used (14). Conventional gradient-echo (GRE) imaging methods (e.g. phase imaging, R2* mapping) have demonstrated increased iron content in the SN of PD patients $(15,16)$, consistent with previous post-mortem studies in PD $(4,5)$. However, there are several limitations in $\mathrm{R} 2^{*}$ mapping and phase imaging. $\mathrm{R} 2 *$ values are susceptible to numerous additional factors, such as the sizes and spacing of inhomogeneities, the rate of water diffusion, field strength and echo times $(17,18)$, and phase is nonlocal and orientation dependent (19). A novel MRI technique, quantitative susceptibility mapping (QSM), overcomes the limitations mentioned above and provides a reliable method to evaluate the iron content in the brain (19-21). A post-mortem study (22) demonstrated a strong linear correlation between the chemically determined iron concentration and the magnetic susceptibility in grey matter structures $(r=0.84, \mathrm{P}<0.001)$ and QSM more effectively depicted deep grey nuclei compared with current standard-of-care sequences (For example, T2-weighted, T2*-weighted, R2* mapping, and phase images) (23).

Increased iron deposition in the SN of PD patients has been confirmed by both biochemical and imaging methods $(4,5,24,25)$. The iron content in other deep grey nuclei (For example, red nucleus and putamen) of PD patients has also been measured, but the results have been conflicting $(25,26)$. In the present study, QSM was utilized to investigate iron homeostasis disorder in PD patients compared with normal controls, while a correlation analysis between observed susceptibility changes and clinical features was conducted in PD patients. To investigate the ability of QSM to measure brain iron, conventional MRI-R2* mapping was also performed in the present study. The diagnostic performances of QSM and R2* mapping to separate PD patients from normal controls were also determined.

\section{Materials and methods}

Subjects. A total of 31 patients diagnosed with idiopathic PD were prospectively recruited from the Neurology Department of The First Affiliated Hospital of Jinan University from July 2017 to December 2018. The patients were diagnosed by two experienced neurologists according to the UK Parkinson's Disease Society Brain Bank clinical diagnostic criteria (27). The following inclusion criteria for patients with PD were used: i) Diagnosis with primary PD; ii) the presence of clear cognitive ability according to a recent clinical assessment; iii) no presence of any other neurological or psychiatric disorder, such as depression or anxiety; and iv) no history of cerebral trauma, cerebrovascular disease or intracranial space-occupying lesions. A total of 6 patients were excluded for poor image quality (e.g. considerable or significant motion artefacts). Ultimately 25 patients with PD were recruited. A total of 28 age- and sex-matched volunteers without a history of neurological disease or brain trauma were recruited as normal controls.

The Hoehn and Yahr (H and Y) score (28) was used by an experienced neurological physician to assess disease severity in the patients with PD. Disease duration was determined from the first occurrence of PD-related symptoms to the date of the study visit. All patients accepted dopaminergic replacement therapy in the present study to improve symptoms. The ethics committee of The First Affiliated Hospital of Jinan University approved the present study, and all subjects provided written informed consent.

MRI data acquisition. All participants underwent MR examinations on a 3.0 T MR scanner (MR750; GE Healthcare) equipped with an 8-channel phased-array head coil. During MRI scanning, foam padding and earplugs were used by the patients to prevent head movement and reduce scanner noise. An axial three-dimensional multi-echo GRE sequence was acquired for the reconstruction of QSM and R2* maps. The following imaging parameters for the multi-echo GRE sequence were used: Repetition time (TR), $49.3 \mathrm{msec}$; ten echoes (echo time, 4.2, 8.8, 13.4, 18.0, 22.7, 27.3, 31.9, $36.5,41.1$ and $45.7 \mathrm{msec}$ ); bandwidth, $62.5 \mathrm{~Hz} /$ pixel; flip angle, 20; field of view, $24 \times 24 \mathrm{~cm}$; matrix, 512x512; resolution, $0.47 \times 0.47 \times 2.0 \mathrm{~mm}^{3}$; acceleration factor, 1 ; and slices, 62. Furthermore, routine MR images, including T1-weighted images, T2-weighted images and T2-weighted fluid-attenuated inversion recovery, were acquired for scanning cerebrovascular diseases.

Image reconstruction. QSM images were reconstructed as previously described (20). In brief, the phase image for each channel of the coil was acquired and fitted; then, phase unwrapping was performed using the Laplacian method $(20,29)$. As the significant background phase was mixed with the true tissue phase contrast, the sophisticated harmonic artefact reduction for phase data was used to remove the background phase before calculating the tissue susceptibility distribution, and the filter radius was set as 8 (30). Finally, the resulting true tissue phase image was used to compute the susceptibility maps using an improved least squares with QR-factorization (LSQR) method (iLSQR) $(20,31)$ with a regularization parameter of 0.04 for Laplace filtering. The magnetic susceptibility distribution map reconstructed by the iLSQR method provided more local details of the brain tissue with negligible streaking artefacts compared to multiple orientation QSM reconstruction (31). 
$\mathrm{R} 2^{*}$ maps were reconstructed using the multi-echo GRE magnitude images in the ADW 4.5 post-processing workstation (GE Healthcare) and quantitative R2* values were calculated with a mono-exponential fit in the Functool 9 platform version 4.5 (GE Healthcare).

Image analysis. The regions of interest (ROIs) were manually added onto the susceptibility maps using ImageJ software 1.46 (National Institutes of Health) by two neuro-radiologists, who were blinded to the information of subjects. The ROIs in the deep grey nucleus covered the bilateral head of the caudate nucleus (CN), SN, red nucleus (RN), globus pallidus (GP) and putamen (PT). The same ROI was applied to obtain R2* values in each region. To minimize measurement errors, the susceptibility and $\mathrm{R} 2^{*}$ values for each nucleus were only obtained from more representative slices in which the ROIs were clearly visible and then the mean of the values from all slices in each region and the bilateral cerebral hemispheres were calculated for subsequent analysis. In the present study, the susceptibility values obtained from the QSM images were used for direct comparison without reference to any selected structure, which is similar to previous studies $(32,33)$.

Statistical analysis. To compare the differences in age and sex distribution between the patients with PD and normal controls, independent samples t-test and the Pearson $\chi^{2}$ test were used. To evaluate the inter-observer reliability of the QSM and $\mathrm{R} 2^{*}$ measurement by two neuro-radiologists, the intra-class correlation coefficient (ICC) was calculated. An ICC $>0.81$ was considered for excellent agreement; 0.61-0.80, good agreement; $0.41-0.60$, moderate agreement; and $<0.40$, poor agreement. The normality of the data was analyzed using the Kolmogorov-Smirnov test. Measurement data were expressed as mean \pm standard deviation. The group differences in the regional susceptibility and $\mathrm{R} 2^{*}$ values between patients with PD and normal controls were compared using an independent samples t-test. When significant differences were found between the two groups, the diagnostic characteristics of QSM and R2* mapping were analyzed for the corresponding region using receiver operating characteristic (ROC) curves. A correlation analysis between susceptibility and $\mathrm{R} 2^{*}$ values and clinical features in patients with PD was performed. Pearson's correlation analysis was also utilized to investigate the correlation between the average susceptibility or $\mathrm{R} 2^{*}$ values in each targeted region for all the normal controls in the present study, and the mean iron concentration in the corresponding regions of normal individuals determined in a previous post-mortem study (13). $\mathrm{P}<0.05$ was considered to indicate a statistically significant difference. SPSS v13.0 (SPSS, Inc.) was used for all statistical analyses.

\section{Results}

Demographic and clinical data assessment. A total of 25 patients (14 males and 11 females; mean age $65.28 \pm 8.32$ years) with $\mathrm{PD}$ and 28 sex- and age-matched volunteers (10 males and 18 females; mean age $63.67 \pm 9.58$ years) were recruited in the present study. No significant differences in age $(\mathrm{P}=0.521)$ or sex $(\mathrm{P}=0.152)$ between the patients with $\mathrm{PD}$ and controls were found.
Table I. Clinical data from patients with Parkinson's disease.

\begin{tabular}{|c|c|c|}
\hline Patient number & $\begin{array}{c}\text { Disease duration, } \\
\text { years }\end{array}$ & $\begin{array}{c}\text { Hoen and Yahr } \\
\text { stage }\end{array}$ \\
\hline 1 & 7 & 3 \\
\hline 2 & 0.33 & 2 \\
\hline 3 & 1 & 1 \\
\hline 4 & 3 & 2 \\
\hline 5 & 3 & 3 \\
\hline 6 & 4 & 1.5 \\
\hline 7 & 2 & 3 \\
\hline 8 & 5 & 1.5 \\
\hline 9 & 4 & 3 \\
\hline 10 & 0.66 & 3 \\
\hline 11 & 3 & 2.5 \\
\hline 12 & 4 & 1.5 \\
\hline 13 & 5 & 3 \\
\hline 14 & 3 & 3 \\
\hline 15 & 5 & 3 \\
\hline 16 & 3 & 2 \\
\hline 17 & 11 & 2 \\
\hline 18 & 10 & 3 \\
\hline 19 & 10 & 2.5 \\
\hline 20 & 2 & 2 \\
\hline 21 & 2 & 2 \\
\hline 22 & 1 & 1.5 \\
\hline 23 & 5 & 2 \\
\hline 24 & 2 & 1.5 \\
\hline 25 & 18 & 3 \\
\hline
\end{tabular}

The primary clinical characteristics in patients with PD include rest tremor, bradykinesia, muscle rigidity and abnormal posture and pace (1). All of the 25 patients with PD had varying degrees of the aforementioned symptoms. Of the 25 cases, 6 had mild symptoms that involved only one limb. A further 19 patients had bilateral limb involvement and 10 had moderate symptoms. The disease duration and the $\mathrm{H}$ and $\mathrm{Y}$ scores for the patients with PD are listed in Table I.

Brain iron distribution in QSM and $R 2^{*}$ mapping. The targeted deep grey matter nuclei in the basal ganglia and midbrain appeared hyper-intense in both QSM and R2* images, and the boundaries of the ROIs appeared clearer in the QSM images compared with that in the R2* images (Fig. 1; images from a patient with PD). Therefore, targeted structures could be manually segmented directly on the QSM images (Fig. 2; images from a normal control).

Interrater agreement. The ICCs of regional magnetic susceptibilities (ICC, 0.977; $\mathrm{P}<0.001$ ) and $\mathrm{R} 2{ }^{*}$ rate constants (ICC, $0.945 ; \mathrm{P}<0.001$ ) between two neuro-radiologists were $>0.81$, revealing excellent inter-rater agreement.

Group differences as assessed by R2* mapping and QSM. The regional magnetic susceptibilities and $\mathrm{R} 2^{*}$ values in the 
Table II. Regional magnetic susceptibilities and R2* values in patients with PD and normal controls.

\begin{tabular}{|c|c|c|c|c|c|c|}
\hline \multirow[b]{2}{*}{ Region of interest } & \multicolumn{3}{|c|}{ Susceptibility, ppm } & \multicolumn{3}{|c|}{$\mathrm{R} 2 *$ value, $\mathrm{sec}^{-1}$} \\
\hline & PD & $\mathrm{NCs}$ & P-value & $\mathrm{PD}$ & $\mathrm{NCs}$ & $\mathrm{P}$-value \\
\hline SN & $0.116 \pm 0.025$ & $0.100 \pm 0.018$ & $<0.001$ & $45.63 \pm 9.32$ & $41.81 \pm 6.08$ & 0.013 \\
\hline $\mathrm{RN}$ & $0.106 \pm 0.022$ & $0.098 \pm 0.018$ & 0.035 & $37.33 \pm 5.71$ & $37.11 \pm 6.51$ & 0.857 \\
\hline $\mathrm{CN}$ & $0.039 \pm 0.015$ & $0.039 \pm 0.011$ & 0.961 & $26.06 \pm 4.89$ & $26.31 \pm 4.84$ & 0.792 \\
\hline PT & $0.034 \pm 0.014$ & $0.042 \pm 0.011$ & 0.002 & $30.36 \pm 6.43$ & $30.62 \pm 5.29$ & 0.817 \\
\hline GP & $0.123 \pm 0.032$ & $0.115 \pm 0.027$ & 0.164 & $51.35 \pm 13.13$ & $48.95 \pm 9.82$ & 0.286 \\
\hline
\end{tabular}

The data are presented as the mean \pm standard deviation. PD, Parkinson's disease; NCs, Normal controls; CN, head of the caudate nucleus, $\mathrm{SN}$, substantia nigra, RN, red nucleus, GP, globus pallidus, PT, putamen.
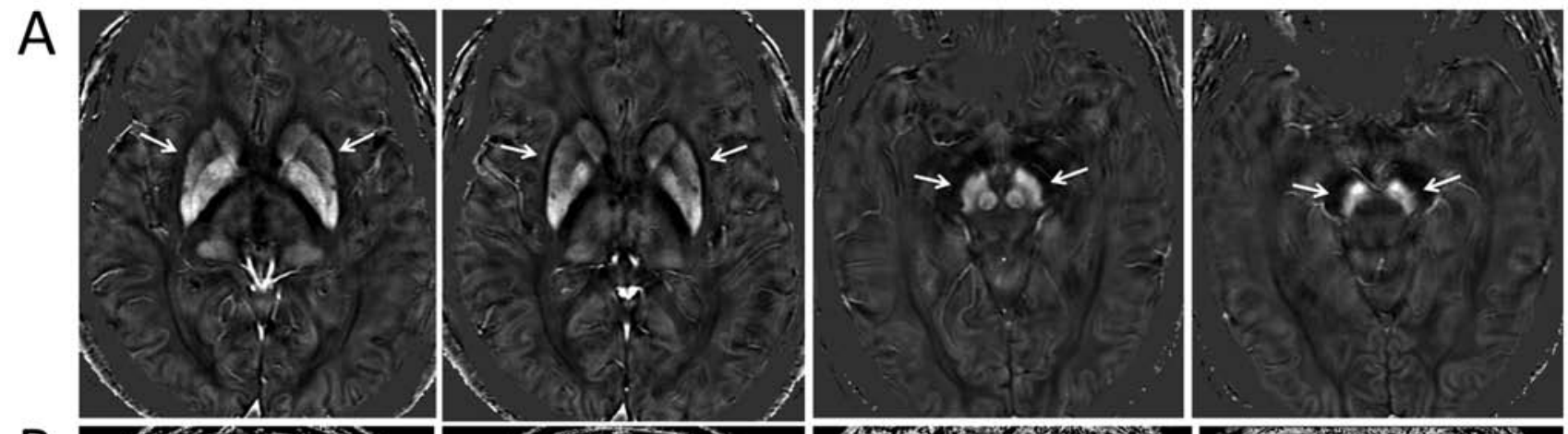

B
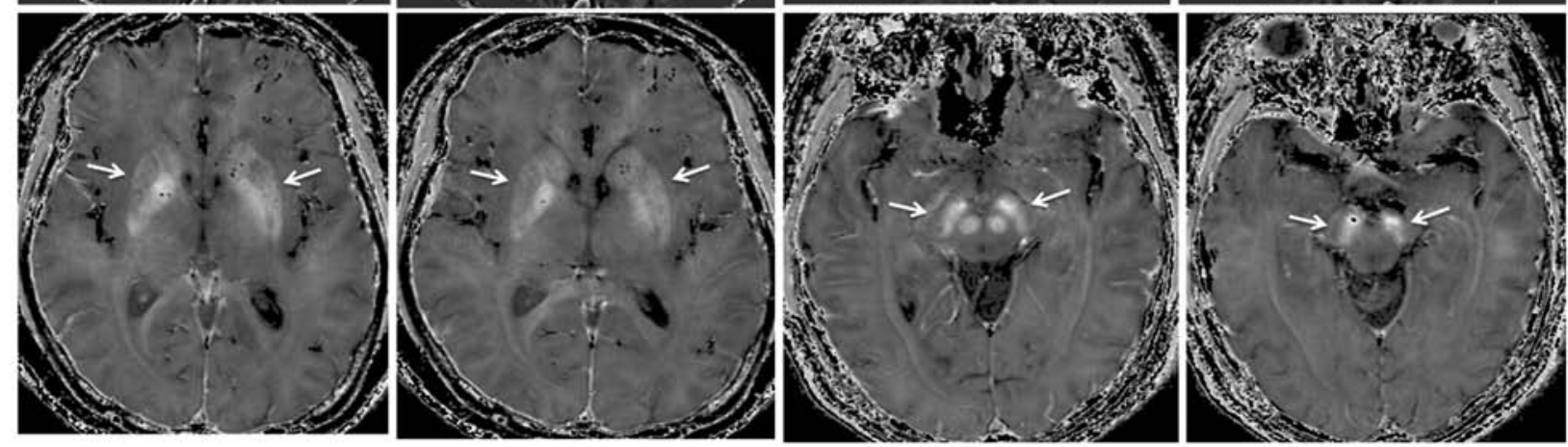

Figure 1. Deep grey nuclei presented on susceptibility and R2* maps. The nuclei in the basal ganglia and midbrain appeared hyper-intense in (A) QSM and (B) R2* maps in a patient with Parkinson's disease. The boundaries of the deep grey matter nuclei are clearer, and the signals are higher in the susceptibility maps.

patients with PD and normal controls were successfully calculated and are listed in Table II. The susceptibility values were significantly higher in the $\mathrm{SN}(\mathrm{P}<0.001)$ and $\mathrm{RN}(\mathrm{P}=0.035)$ in the patients with $\mathrm{PD}$ compared with that in the controls (Fig. 3). However, the susceptibility values in the PT were significantly lower in the patients with $\mathrm{PD}(\mathrm{P}=0.002$; Fig. 3$)$. No significant differences in magnetic susceptibilities were observed in any other region between the patients with PD and normal controls. Increased $\mathrm{R} 2^{*}$ values were observed only in the $\mathrm{SN}$ of the patients with PD compared with that in the controls ( $\mathrm{P}=0.013$; Fig. 4).

Diagnostic performance of QSM and R2* mapping to classify $P D$ patients and normal controls. The abilities of QSM and $\mathrm{R} 2^{*}$ mapping to classify patients with $\mathrm{PD}$ and normal controls were analyzed in the SN, RN and PT using ROC curves (Fig. 5).
The area under the curve and respective standard deviations are shown in Fig. 5. The QSM (SN area, 0.72 \pm 0.05 ; RN area, $0.61 \pm 0.05$; PT area, $0.68 \pm 0.06$ ) had higher sensitivity in classifying patients with PD compared with that for R2* mapping (SN area, 0.63 \pm 0.05 ; RN area, 0.51 \pm 0.06 ; PT area, $0.53 \pm 0.06$ ).

Regional susceptibility and $R 2^{*}$ values correlate with brain iron levels. Significant positive correlations were observed between the regional susceptibility values and the iron concentrations $\left(\mathrm{R}^{2}=0.92 ; \mathrm{P}=0.009\right)$ as well as the $\mathrm{R} 2 *$ values and the iron concentrations $\left(\mathrm{R}^{2}=0.86 ; \mathrm{P}=0.023\right)$ in normal individuals (Data not shown). The iron concentrations were referenced to the Hallgren study (13), who measured the iron concentrations with biochemical methods in normal individuals. This finding indicated that QSM and R2* mapping techniques accurately calculated the regional iron levels in the human brain. 

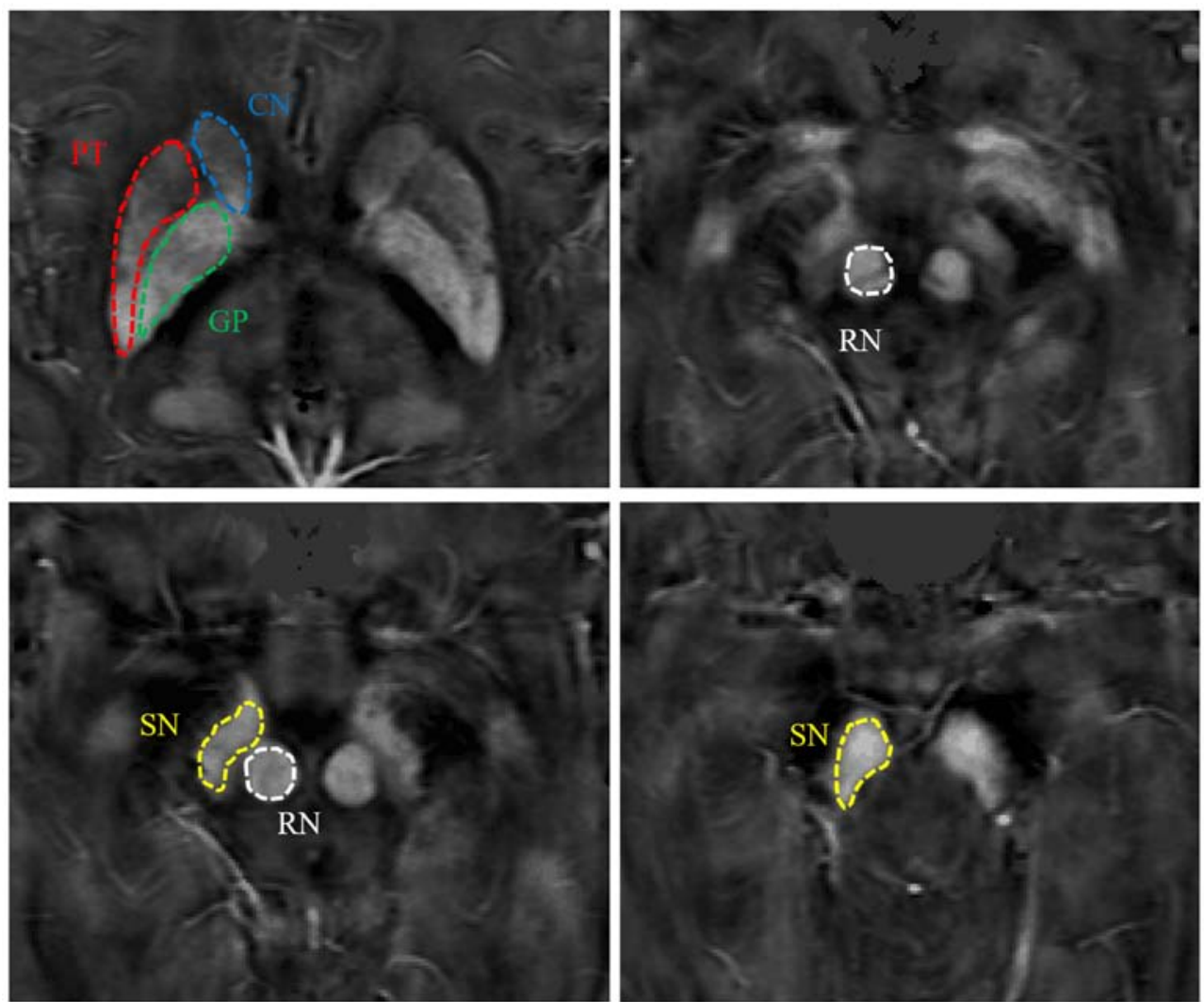

Figure 2. Manual segmentation of ROIs on the susceptibility maps of a 55-year-old normal control. The ROIs covered the head of the CN, SN, RN, GP and PT. $\mathrm{CN}$, caudate nucleus; SN, substantia nigra; RN, substantia nigra; GP, globus pallidus; PT, putamen; ROI, region of interest.

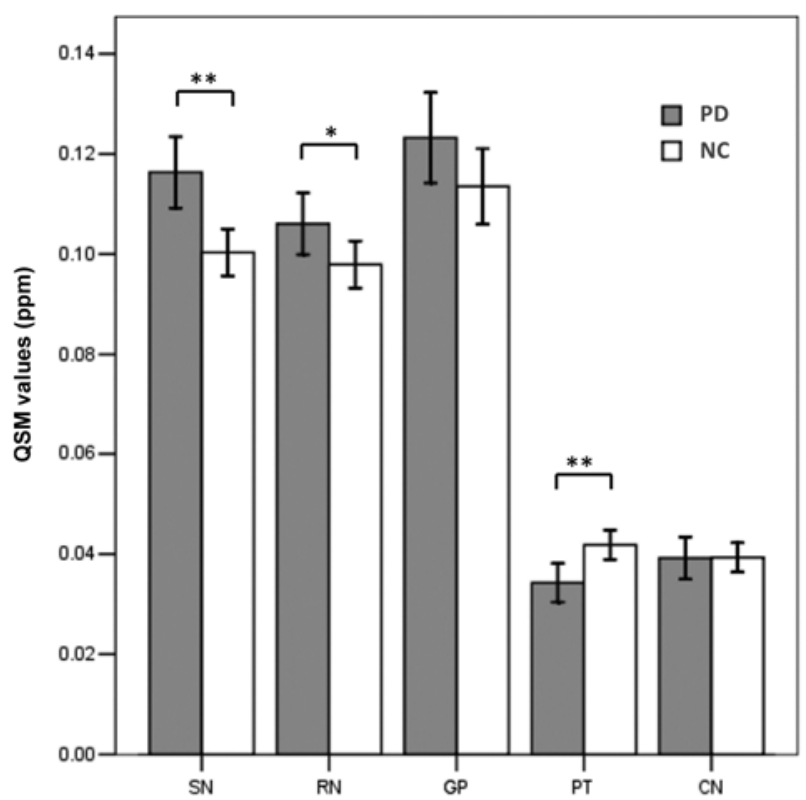

Figure 3. Bar chart of QSM values in all regions of interest in the patients with PD and normal controls. The P-values were calculated using independent samples t-tests. ${ }^{*} \mathrm{P}<0.05,{ }^{* *} \mathrm{P}<0.005$. PD, Parkinson's disease; $\mathrm{NCs}$, Normal controls; $\mathrm{CN}$, head of the caudate nucleus; $\mathrm{SN}$, substantia nigra; $\mathrm{RN}$, red nucleus; GP, globus pallidus; PT, putamen; QSM, quantitative susceptibility mapping.

Correlations of QSM and of $R 2^{*}$ mapping with clinical feature. No significant correlations between the imaging parameters (e.g., susceptibility and $\mathrm{R} 2{ }^{*}$ values) and clinical

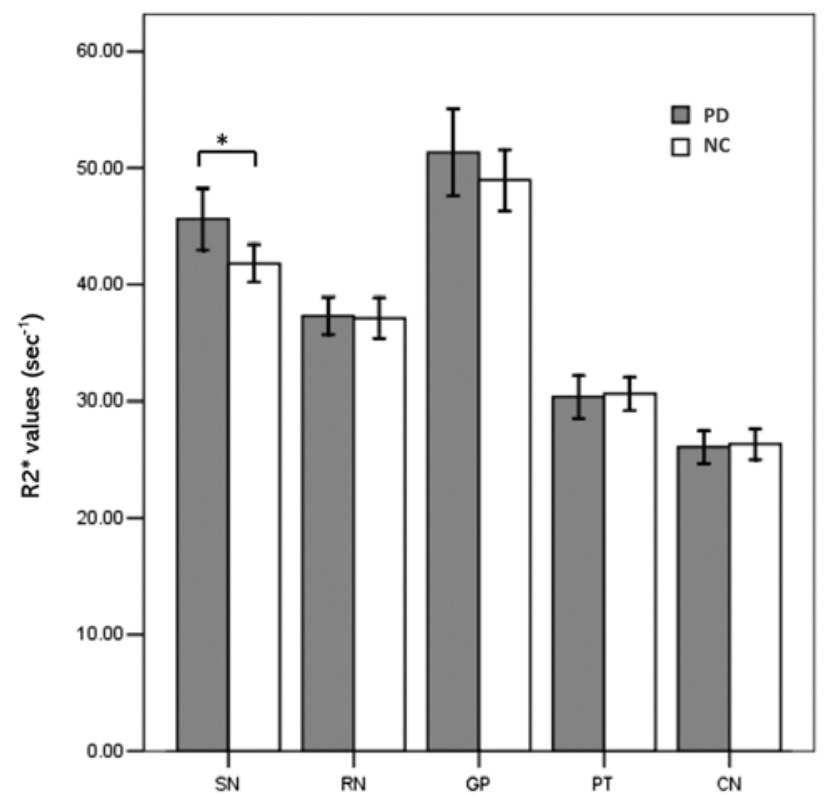

Figure 4. Bar chart of $\mathrm{R} 2 *$ values in all regions of interest in the patients with $\mathrm{PD}$ and normal controls. The P-values were calculated using independent samples t-tests. ${ }^{*} \mathrm{P}<0.05$. PD, Parkinson's disease; NCs, Normal controls; $\mathrm{CN}$, head of the caudate nucleus; $\mathrm{SN}$, substantia nigra; $\mathrm{RN}$, red nucleus; GP, globus pallidus; PT, putamen.

features described by disease durations and the $\mathrm{H}$ and $\mathrm{Y}$ stages were found in the patients with PD in any targeted region $(\mathrm{P}>0.10)$. 

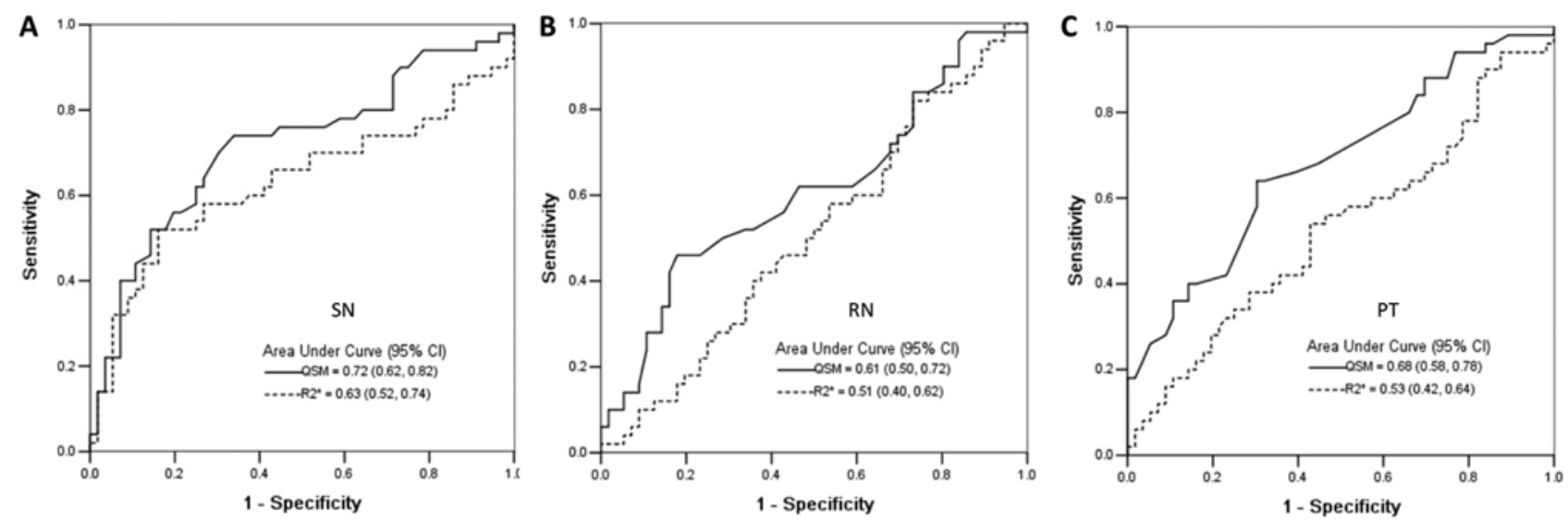

Figure 5. Receiver operating characteristic curve for quantitative susceptibility mapping and R2* mapping in the SN (A) RN (B) and PT (C) SN, substantia nigra; RN, red nucleus; PT, putamen; 95\% CI, 95\% Confidence Interval.

\section{Discussion}

PD is a common neurodegenerative disease and its pathogenesis is still unclear $(2,10)$. A review published in 2006 reported that the prevalence of PD increased with age, and that PD affects $1 \%$ of the population $>60$ years old (2). One of the major pathological changes in PD is iron deposition in the SN (4). Iron protein and haemosiderosis in the brain are strong paramagnetic substances that cause a strong reduction in $\mathrm{T} 2^{*}$ relaxation time (34). Therefore, conventional GRE imaging approaches (magnitude-, phase-, and R2*-imaging) have been used to assess iron changes in the brains of patients with PD. The most commonly used method is R2* mapping, which can be used for quantitative measurement $(15,18,35)$. QSM, as a novel technique, overcomes several non-local restrictions of susceptibility-weighted and phase imaging (For example, the nonlocality of the magnetic field distribution) and provides a non-invasive and quantitative method to assess diseases characterized by variations in iron and/or myelin concentrations $(25,36)$.

Elevated iron deposition in the SN of patients with PD compared with that in controls was demonstrated by QSM in the present study. Increased iron accumulation in the $\mathrm{SN}$ is one of the major pathological changes that occurs in patients with PD and has been reported in both post-mortem $(4,5,37)$ and MRI studies $(25,26,38)$. Another major pathological feature in patients with PD is a loss of dopaminergic neurons in the SN (4). Neuromelanin (NM), a dark-coloured pigment produced in the dopaminergic neurons of the human $\mathrm{SN}$, has the ability to bind a variety of metals, including iron, and increased NM-bound iron has previously been reported in the SN of patients with PD $(39,40)$. The biochemical mechanisms causing this phenomenon in PD are unclear; however, it is hypothesized that redox-active iron could be released under conditions of oxidative stress and high concentrations of reactive iron lead to further oxidative damage, which induces neuronal vulnerability $(41,42)$.

Elevated susceptibility values were also observed in the RN of patients with PD, which is consistent with previous studies $(24,26)$. A previous study identified higher R2* values in both the SN and RN (34). The RN, located in the midbrain near the SN, is similarly iron-rich (43) and is easily recognized on QSM and R2* images. The $\mathrm{RN}$ receives significant somatotopically organized input from the ipsilateral motor cortex and contralateral cerebellum (44), thus serving as an essential intersection between primary and cerebellar motor pathways $(45,46)$. Lewis et al $(34)$ reported that as an integral part of cerebellar circuitry, the RN might mediate PD-related compensatory changes and the occurrence of dyskinesia. The underlying biochemical mechanism for the increased susceptibility values in the RN remains unclear. It is hypothesized that this mechanism may be similar to that for the $\mathrm{SN}$, due to increased iron deposition in patients with PD. Further histopathological and imaging studies are required to examine the role of iron deposition in the $\mathrm{RN}$ in the pathogenesis of PD.

Notably, reduced susceptibility values in the PT were observed in patients with PD when compared with that in the controls. A consistently reduced iron level in the PT of patients with PD has been reported in both post-mortem and MRI studies $(5,47)$. However, another study demonstrated increased iron deposition in the PT of patients with PD (4), and numerous additional studies did not find a significant difference in iron levels in the PT between patients with PD and healthy controls $(5,24)$. Variations in the iron levels in sub-regions of the PT and iron migration, through unclear pathways between different brain regions during the progression of PD may be the causes of these contradictory findings. Moreover, different methods (For example, atomic absorption spectrophotometry, R2* mapping and QSM) for quantifying brain iron levels and the heterogeneity of patients with PD in these studies may also be sources of this discrepancy.

In the present study, increased $\mathrm{R} 2{ }^{*}$ values were only observed in the SN of the patients with PD compared with that in the controls and previous studies $(25,26,34)$ have demonstrated consistent findings. The increased iron deposition in the $\mathrm{SN}$ of patients with PD has been confirmed by previous studies $(4,37)$. Iron is paramagnetic and causes a strong increase in R2* relaxation rates (48). Therefore, it is feasible to distinguish PD patients from healthy controls using R2* mapping. The difference in $\mathrm{R} 2 *$ values in other regions was not observed between the PD patients and normal controls. This finding may indicate that R2* mapping is less sensitive than QSM to iron changes in the brains of PD patients. The $\mathrm{R} 2$ * relaxation rate is affected not only by iron but also by numerous additional factors, such as the size and spacing of 
the inhomogeneities, the rate of water diffusion, field strength and echo times $(17,18)$. These factors will reduce the sensitivity and accuracy of R2* mapping in detecting susceptibility changes caused by iron (25).

The regional susceptibility values were positively correlated with the iron concentrations determined in previous post-mortem studies (13) of healthy individuals $(\mathrm{r}=0.957$; $\mathrm{P}=0.011$ ). Similarly, a post-mortem QSM study (22) also demonstrated a strong linear correlation between chemically determined iron concentrations and magnetic susceptibility in grey matter structures $(r=0.84 ; \mathrm{P}<0.001)$. This finding confirmed that the QSM was sensitive and reliable for assessing the iron level in the human brain. A positive correlation between $\mathrm{R} 2^{*}$ values and iron concentrations (13) was also observed $(\mathrm{r}=0.904 ; \mathrm{P}=0.035)$, but this correlation was slightly weaker compared with the correlation between susceptibility values and iron concentrations. The targeted deep grey matter nuclei in the basal ganglia and midbrain appeared hyper-intense in both QSM and R2* images, and the boundary of the ROIs was clearer in QSM maps, consistent with a previous QSM study (23). These findings indicate that QSM can detect iron levels and depict the deep grey nuclei in the human brain more effectively compared with $\mathrm{R} 2{ }^{*}$ mapping.

To date, only two studies $(49,50)$ have analyzed the abilities of QSM and R2* mapping to classify healthy individuals and patients with PD in the SN using the ROC curve, which displayed areas under the curve of 0.77 and 0.54 for QSM and $\mathrm{R} 2{ }^{*}$ mapping (50) and an area under the curve of 0.72 for R2* mapping (49). In the present study, the ROC analysis displayed an area under the curve of 0.72 and 0.63 for QSM and R2* mapping in the SN. The diagnostic characteristics of QSM and $\mathrm{R} 2^{*}$ mapping in the $\mathrm{RN}$ and $\mathrm{PT}$ were further investigated in addition to the $\mathrm{SN}$, as the susceptibility values in the RN and PT were also significantly different between patients with PD and normal controls. To the best of our knowledge, this is the first time that the diagnostic characteristics of QSM and $\mathrm{R} 2^{*}$ mapping in the RN and PT have been investigated. The QSM showed higher sensitivity to classify patients with PD compared with that for $\mathrm{R} 2^{*}$ mapping for all regions analyzed. The area under the curve for QSM and $\mathrm{R} 2^{*}$ mapping in the $\mathrm{SN}$ was also found to be higher compared with that in the RN and PT.

There were no significant correlations between the imaging parameters in all targeted regions and clinical features described by disease duration and the $\mathrm{H}$ and $\mathrm{Y}$ stages in the patients with PD. These results suggest that in patients with different $\mathrm{H}$ and $\mathrm{Y}$ stages, and disease durations, the changes in iron levels were not significant. Previous studies $(16,50)$ also did not find a significant correlation between the regional iron levels and disease durations. He et al (26) reported a positive correlation between increased susceptibility values in the SN and disease duration in patients with PD, but the elevated R2* values in the $\mathrm{SN}$ were not correlated with clinical features. Langkammer et al (24) demonstrated that the SN susceptibility values were correlated with $\mathrm{H}$ and $\mathrm{Y}$ stages but were not correlated with disease duration in patients with PD. However, the aforementioned correlation between increased iron levels in the SN and the clinical features in patients with $\mathrm{PD}$ was low $(\mathrm{r}=0.29)$. Therefore, it is debatable whether it is reliable to evaluate the progression of PD using QSM or R2* mapping.

There are several limitations to the present study. First, the sample size was small, so a contrast analysis between patients with early-stage and advanced-stage PD was not performed. PD is asymmetrical and patients with early-stage PD often suffer from unilateral limb involvement (26). As the number of patients with early-stage PD was limited, a contrast analysis between the ipsilateral and the contralateral regions was not performed. In future studies, more patients with early-stage PD will be recruited and contrast analysis between the ipsilateral and the contralateral regions will be performed. Previous studies (51-53) separated the SN into pars compacta and pars reticulata with ultra high-field, 7 Tesla MRI and showed iron deposition was primarily located in the pars compacta; however, in the present study, the SN was evaluated as a whole to analyse the clinical GRE data. Another limitation of the present study is the lack of pathological confirmation in the patients with PD. However, all patients who may clinically have idiopathic PD undergo a longitudinal clinical assessment and their assessment is reassessed over time.

In conclusion, the results indicate that both QSM and R2* mapping are feasible techniques to calculate iron levels in human brains, although QSM provides a more sensitive and accurate method to assess regional abnormal iron distribution in patients with PD. QSM has a higher sensitivity in the classification of patients with PD from normal controls compared with that in $\mathrm{R} 2{ }^{*}$ mapping.

\section{Acknowledgements}

Not applicable.

\section{Funding}

The present study was supported by Department of science and Technology of Guangdong province [grant. nos. 2014A020212689].

\section{Availability of data and materials}

The datasets used and/or analyzed during the current study are available from the corresponding author on reasonable request.

\section{Authors' contributions}

LL and CS contributed to the design and conception of the study. JL and XL designed MR inspection sequence parameters and participated in implementation inspections. QC, $\mathrm{JH}$, and MM obtained, analyzed and interpreted the data. QC and $\mathrm{JH}$ were responsible for the writing of the manuscript. $\mathrm{JL}$ and XL reviewed the manuscript and critically revised important intellectual content. QZ was in charge of the statistical analysis. All authors agreed to be accountable for all aspects of the work in ensuring that questions related to the accuracy or integrity of any part of the work are appropriately investigated and resolved. All authors read and approved the final version. 


\section{Ethics approval and consent to participate}

The Ethics Committee of The First Affiliated Hospital of Jinan University approved the present study, and all subjects provided written informed consent.

\section{Patient consent for publication}

Not applicable.

\section{Competing interests}

The authors declare that they have no competing interests.

\section{References}

1. Marino BLB, Souza LR, Sousa KPA, Ferreira JV, Padilha EC, Silva CHTP, Taft CA and Hage-Melim LIS: Parkinson's disease: A Review from the Pathophysiology to Diagnosis, New Perspectives for Pharmacological Treatment. Mini Rev Med Chem: Nov 3, 2019 (Epub ahead of print). doi: 10.2174/13895575 19666191104110908.

2. de Lau LM and Breteler MM: Epidemiology of Parkinson's disease. Lancet Neurol 5: 525-535, 2006.

3. Fasano M, Bergamasco B and Lopiano L: Modifications of the iron-neuromelanin system in Parkinson's disease. J Neurochem 96: 909-916, 2006.

4. Griffiths PD and Crossman AR: Distribution of iron in the basal ganglia and neocortex in postmortem tissue in Parkinson's disease and Alzheimer's disease. Dementia 4: 61-65, 1993.

5. Dexter DT, Carayon A, Javoy-Agid F, Agid Y, Wells FR, Daniel SE, Lees AJ, Jenner P and Marsden CD: Alterations in the levels of iron, ferritin and other trace metals in Parkinson's disease and other neurodegenerative diseases affecting the basal ganglia. Brain 114: 1953-1975, 1991.

6. Dusek P, Jankovic J and Le W: Iron dysregulation in movement disorders. Neurobiol Dis 46: 1-18, 2012.

7. Rhodes SL, Buchanan DD, Ahmed I, Taylor KD, Loriot MA, Sinsheimer JS, Bronstein JM, Elbaz A, Mellick GD, Rotter JI and Ritz B: Pooled analysis of iron-related genes in Parkinson's disease: Association with transferrin. Neurobiol Dis 62: 172-178, 2014.

8. Sian-Hulsmann J, Mandel S, Youdim MB and Riederer P: The relevance of iron in the pathogenesis of Parkinson's disease. J Neurochem 118: 939-957, 2011.

9. Andersen JK: Oxidative stress in neurodegeneration: Cause or consequence? Nat Med 10 (Suppl): S18-S25, 2004.

10. Tysnes OB and Storstein A: Epidemiology of Parkinson's disease. J Neural Transm (Vienna) 124: 901-905, 2017.

11. Hardy PA, Gash D, Yokel R, Andersen A, Ai Y and Zhang Z: Correlation of R2 with total iron concentration in the brains of rhesus monkeys. J Magn Reson Imaging 21: 118-127, 2005.

12. Aquino D, Bizzi A, Grisoli M, Garavaglia B, Bruzzone MG, Nardocci N, Savoiardo M and Chiapparini L: Age-related iron deposition in the basal ganglia: Quantitative analysis in healthy subjects. Radiology 252: 165-172, 2009.

13. Hallgren B and Sourander P: The effect of age on the non-haemin iron in the human brain. J Neurochem 3: 41-51, 1958.

14. Ropele S, Wattjes MP, Langkammer C, Kilsdonk ID, Graaf WL, Frederiksen JL, Fuglø D, Yiannakas M, Wheeler-Kingshott CA, Enzinger C, et al: Multicenter R2* mapping in the healthy brain. Magn Reson Med 71: 1103-1107, 2014.

15. Du G, Lewis MM, Styner M, Shaffer ML, Sen S, Yang QX and Huang X: Combined R2* and diffusion tensor imaging changes in the substantia nigra in Parkinson's disease. Mov Disord 26: 1627-1632, 2011.

16. Martin WR, Wieler M and Gee M: Midbrain iron content in early Parkinson disease: A potential biomarker of disease status. Neurology 70: 1411-1417, 2008.

17. Kennan RP, Zhong J and Gore JC: Intravascular susceptibility contrast mechanisms in tissues. Magn Reson Med 31: 9-21, 1994.

18. Fernandez-Seara MA and Wehrli FW: Postprocessing technique to correct for background gradients in image-based $\mathrm{R}^{*}(2)$ measurements. Magn Reson Med 44: 358-366, 2000.
19. Liu C, Li W, Tong KA, Yeom KW and Kuzminski S: Susceptibility-weighted imaging and quantitative susceptibility mapping in the brain. J Magn Reson Imaging 42: 23-41, 2015.

20. Li W, Wu B and Liu C: Quantitative susceptibility mapping of human brain reflects spatial variation in tissue composition. NeuroImage 55: 1645-1656, 2011.

21. Liu T, Spincemaille P, de Rochefort L, Kressler B and Wang Y: Calculation of susceptibility through multiple orientation sampling (COSMOS): A method for conditioning the inverse problem from measured magnetic field map to susceptibility source image in MRI. Magn Reson Med 61: 196-204, 2009.

22. Langkammer C, Schweser F, Krebs N, Deistung A, Goessler W, Scheurer E, Sommer K, Reishofer G, Yen K, Fazekas F, et al: Quantitative susceptibility mapping (QSM) as a means to measure brain iron? A post mortem validation study. NeuroImage 62: 1593-1599, 2012.

23. Liu T, Eskreis-Winkler S, Schweitzer AD, Chen W, Kaplitt MG, Tsiouris AJ and Wang Y: Improved subthalamic nucleus depiction with quantitative susceptibility mapping. Radiology 269: 216-223, 2013.

24. Langkammer C, Pirpamer L, Seiler S, Deistung A, Schweser F, Franthal S, Homayoon N, Katschnig-Winter P, Koegl-Wallner M, Pendl T, et al: Quantitative Susceptibility Mapping in Parkinson's Disease. PLoS One 11: e0162460, 2016.

25. Murakami Y, Kakeda S, Watanabe K, Ueda I, Ogasawara A, Moriya J, Ide S, Futatsuya K, Sato T, Okada K, et al: Usefulness of quantitative susceptibility mapping for the diagnosis of Parkinson disease. AJNR Am J Neuroradiol 36: 1102-1108, 2015.

26. He N, Ling H, Ding B, Huang J, Zhang Y, Zhang Z, Liu C, Chen $\mathrm{K}$ and Yan F: Region-specific disturbed iron distribution in early idiopathic Parkinson's disease measured by quantitative susceptibility mapping. Hum Brain Mapp 36: 4407-4420, 2015.

27. Hughes AJ, Daniel SE, Kilford L and Lees AJ: Accuracy of clinical diagnosis of idiopathic Parkinson's disease: A clinico-pathological study of 100 cases. J Neurol Neurosurg Psychiatry 55: 181-184, 1992.

28. Hoehn MM and Yahr MD: Parkinsonism: Onset, progression, and mortality. 1967. Neurology 57 (10 Suppl 3): S11-S26, 2001.

29. Wu B, Li W, Guidon A and Liu C: Whole brain susceptibility mapping using compressed sensing. Magn Reson Med 67: $137-147,2012$.

30. Schweser F, Deistung A, Lehr BW and Reichenbach JR: Quantitative imaging of intrinsic magnetic tissue properties using MRI signal phase: An approach to in vivo brain iron metabolism? NeuroImage 54: 2789-2807, 2011.

31. Li W, Wang N, Yu F, Han H, Cao W, Romero R, Tantiwongkosi B, Duong TQ and Liu C: A method for estimating and removing streaking artifacts in quantitative susceptibility mapping. NeuroImage 108: 111-122, 2015.

32. Li W, Langkammer C, Chou YH, Petrovic K, Schmidt R, Song AW, Madden DJ, Ropele S and Liu C: Association between increased magnetic susceptibility of deep gray matter nuclei and decreased motor function in healthy adults. NeuroImage 105: 45-52, 2015.

33. Li W, Wu B, Batrachenko A, Bancroft-Wu V, Morey RA, Shashi V, Langkammer C, De Bellis MD, Ropele S, Song AW and Liu C: Differential developmental trajectories of magnetic susceptibility in human brain gray and white matter over the lifespan. Hum Brain Mapp 35: 2698-2713, 2014.

34. Lewis MM, Du G, Kidacki M, Patel N, Shaffer ML, Mailman RB and Huang X: Higher iron in the red nucleus marks Parkinson's dyskinesia. Neurobiol Aging 34: 1497-1503, 2013.

35. Zywicke HA, van Gelderen P, Connor JR, Burdo JR, Garrick MD, Dolan KG, Frank JA and Bulte JW: Microscopic R2* mapping of reduced brain iron in the Belgrade rat. Ann Neurol 52: 102-105, 2002.

36. Liu C, Wei H, Gong NJ, Cronin M, Dibb R and Decker K: Quantitative susceptibility mapping: Contrast mechanisms and clinical applications. Tomography 1: 3-17, 2015.

37. Griffiths PD, Dobson BR, Jones GR and Clarke DT: Iron in the basal ganglia in Parkinson's disease. An in vitro study using extended X-ray absorption fine structure and cryo-electron microscopy. Brain 122: 667-673, 1999.

38. Meineke J, Rauscher A, Marques JP, Bilgic B and Langkammer C: Quantitative Susceptibility Mapping in Parkinson's Disease. Magnetic Resonance Med 11: e0162460, 2016. 
39. Double KL, Gerlach M, Youdim MB and Riederer P: Impaired iron homeostasis in Parkinson's disease. J Neural Transm Suppl: S37-S58, 2000.

40. Gerlach M, Double KL, Ben-Shachar D, Zecca L, Youdim MB and Riederer P: Neuromelanin and its interaction with iron as a potential risk factor for dopaminergic neurodegeneration underlying Parkinson's disease. Neurotox Res 5: 35-44, 2003.

41. Zecca L, Youdim MB, Riederer P, Connor JR and Crichton RR: Iron, brain ageing and neurodegenerative disorders. Nat Rev Neurosci 5: 863-873, 2004.

42. Gerlach M, Double KL, Youdim MB and Riederer P: Potential sources of increased iron in the substantia nigra of parkinsonian patients. J Neural Transm Suppl: S133-S142, 2006.

43. Drayer B, Burger P, Darwin R, Riederer S, Herfkens R and Johnson GA: MRI of brain iron. AJR Am J Roentgenol 147: 103-110, 1986.

44. Habas $\mathrm{C}$ and Cabanis EA: Cortical projection to the human red nucleus: Complementary results with probabilistic tractography at 3 T. Neuroradiology 49: 777-784, 2007.

45. Lapresle J and Hamida MB: The dentato-olivary pathway. Somatotopic relationship between the dentate nucleus and the contralateral inferior olive. Arch Neurol 22: 135-143, 1970.

46. Bird TD and Shaw CM: Progressive myoclonus and epilepsy with dentatorubral degeneration: A clinicopathological study of the Ramsay Hunt syndrome. J Neurol Neurosurg Psychiatry 41: 140-149, 1978

47. Graham JM, Paley MN, Grunewald RA, Hoggard N and Griffiths PD: Brain iron deposition in Parkinson's disease imaged using the PRIME magnetic resonance sequence. Brain 123 Pt 12: 2423-2431, 2000.
48. Wood JC, Enriquez C, Ghugre N, Tyzka JM, Carson S, Nelson MD and Coates TD: MRI R2 and R2* mapping accurately estimates hepatic iron concentration in transfusion-dependent thalassemia and sickle cell disease patients. Blood 106: 1460-1465, 2005.

49. Homayoon N, Pirpamer L, Franthal S, Katschnig-Winter P, Kögl M, Seiler S, Wenzel K, Hofer E, Deutschmann H, Fazekas F, et al: Nigral iron deposition in common tremor disorders. Mov Disord 34: 129-132, 2019.

50. Barbosa JH, Santos AC, Tumas V, Liu M, Zheng W, Haacke EM and Salmon CE: Quantifying brain iron deposition in patients with Parkinson's disease using quantitative susceptibility mapping, R2 and R2. Magn Reson Imaging 33: 559-565, 2015.

51. Lotfipour AK, Wharton S, Schwarz ST, Gontu V, Schäfer A, Peters AM, Bowtell RW, Auer DP, Gowland PA and Bajaj NP: High resolution magnetic susceptibility mapping of the substantia nigra in Parkinson's disease. J Magn Reson Imaging 35: 48-55, 2012.

52. Eapen M, Zald DH, Gatenby JC, Ding Z and Gore JC: Using high-resolution MR imaging at $7 \mathrm{~T}$ to evaluate the anatomy of the midbrain dopaminergic system. AJNR Am J Neuroradiol 32: 688-694, 2011.

53. Schafer A, Forstmann BU, Neumann J, Wharton S, Mietke A, Bowtell R and Turner R: Direct visualization of the subthalamic nucleus and its iron distribution using high-resolution susceptibility mapping. Hum Brain Mapp 33: 2831-2842, 2012.

(i) (5) $\ominus$ This work is licensed under a Creative Commons Attribution-NonCommercial-NoDerivatives 4.0 International (CC BY-NC-ND 4.0) License. 